\title{
Impacto da autopercepção de saúde em longevos com incontinência urinária
}

\author{
Impact of self-perceived health on long-lived subjects with urinary incontinence
}

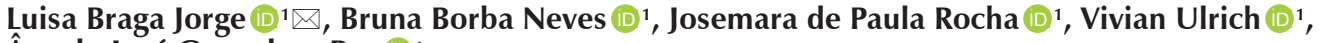 \\ Ângelo José Gonçalves Bos (D) 1
}

\author{
Programa de Pós-graduação em Gerontologia Biomédica, Escola de Medicina, Pontifícia Universidade Católica do Rio Grande do Sul. Porto Alegre, \\ Rio Grande do Sul, Brasil.
}

Como citar este artigo (How to cite this article):

Jorge LB, Neves BB, Rocha JP, Ulrich V, Bos AJG. Impacto da autopercepção de saúde em longevos com incontinência urinária (Impact of self-perceived health on long-lived subjects with urinary incontinence). Sci Med. 2019;29(1):e32831. https://doi.org/10.15448/19806108.2019 .1 .32831

\section{RESUMO}

OBJETIVOS: Verificar o impacto da autopercepção de saúde sobre a chance de desenvolver incontinência urinária em longevos.

MÉTODOS: Trata-se de um estudo transversal e analítico com idosos de 90 anos ou mais. Foi realizada análise estatística analítica e descritiva (frequências, média e desvio padrão, análise de regressão logística univariada e ajustada pela autopercepção de saúde) das variáveis sociodemográficas e clínicas (sexo, estado conjugal, continência, idade, sintomas depressivos, cognição, comorbidades e facilidade de realizar atividades básicas e funcionais).

RESULTADOS: Participaram 182 longevos, 71\% mulheres. O diagnóstico prévio de depressão, o escore de sintomas depressivos e o percentual de facilidade para atividades funcionais e básicas foram relacionados com a autopercepção de saúde $(p<0,05)$. A perda de urina, contudo, não foi relacionada à autopercepção de saúde. A autopercepção de saúde influenciou o efeito das variáveis diagnóstico prévio de depressão, número de sintomas depressivos, pontuação do Mini Exame do Estado Mental e número de comorbidades sobre a chance de incontinência urinária na presença das mesmas. O sexo masculino, a viuvez e a facilidade no desempenho de atividades básicas e funcionais foram variáveis significativamente relacionadas com a incontinência urinária independentemente do ajuste da autopercepção de saúde.

CONCLUSÕES: Não houve relação entre incontinência urinária e autopercepção de saúde, contudo, a autopercepção de saúde influenciou no efeito das variáveis sociodemográficas e clínicas sobre a chance de ter incontinência urinária.

DESCRITORES: idosos; nonagenários; incontinência urinária; autopercepção; qualidade de vida.

\section{ABSTRACT}

AIMS: To verify the impact of self-perceived health on the chance of developing urinary incontinence in very-old.

METHODS: This is a cross-sectional and analytical study with elderly individuals aged 90 years and over. The statistical and analytical variables (sex, marital status, continence, age, depressive symptoms, cognition, comorbidities and ability to perform activities) were analyzed analytical and descriptive statistics (frequencies, mean and standard deviation, univariate and health self-perception adjusted logistic regression analysis). RESULTS: There were 182 participants, $71 \%$ women. The previous diagnosis of depression, the score of depressive symptoms and the percentage of ease for functional and basic activities were related to self-perception of health $(\mathrm{p}<0.05)$. The loss of urine, however, was not related to self-perceived health. The self-perception of health influenced the effect of the variables previous diagnosis of depression, number of depressive symptoms, Mini-Mental State Examination score and number of comorbidities on the chance of urinary incontinence in the presence of these variables. Males, widowhood and ease of performance of basic and functional activities were significantly related to urinary incontinence regardless of the adjustment of health self-perception.

CONCLUSIONS: There was no relationship between urinary incontinence and health self-perception. However, health self-perception influenced the effect of sociodemographic and clinical variables on the chance of having urinary incontinence.

KEYWORDS: elderly; nonagenarian; urinary incontinence; self concept; quality of life. 
Abreviaturas: ABVD, atividades básicas de vida diária; AMPAL, Atenção Multiprofissional ao Longevo; APS, autopercepção de saúde; GDS5, Escala de Depressão Geriátrica 5 (Geriatric Depression Scale 5); IU, incontinência urinária; MEEM, Mini Exame do Estado Mental; QV, qualidade de vida.

\section{INTRODUÇÃO}

Com o aumento da expectativa de vida, a proporção de pessoas com 80 anos ou mais aumentou consideravelmente, sendo as faixas etárias longevas as que mais crescem [1]. Esse aumento da população longeva gera expectativas de maior número de comorbidades, que desafiarão o sistema de saúde [2].

Dentre os gigantes da geriatria que causam importantes repercussões na vida dos idosos, está a incontinência urinaria (IU), muito prevalente nessa população, predispondo aos quadros de fragilidade, complicando o processo de envelhecimento, reduzindo a capacidade funcional, interferindo na participação social e piorando a qualidade de vida (QV) [3]. O conceito de QV inclui a autoestima e o bem-estar pessoal, englobando aspectos relacionados à capacidade funcional, nível socioeconômico, estado emocional, vida social, valores éticos, culturais e religiosos, atividade intelectual, autocuidado, apoio familiar, número de comorbidades e satisfação com a própria vida [4].

A autopercepção de saúde (APS) tem se mostrado um bom indicador do estado de saúde. Trata-se de uma referência para avaliar o bem-estar pessoal vinculado à capacidade de realizar as atividades, ao declínio funcional, à prevalência de doenças crônicas e à mortalidade [5]. Da mesma forma, constitui-se numa ferramenta para detectar as condições e o acesso à saúde por parte da população idosa. Ações que abordam os principais problemas determinantes da APS contribuem para a promoção de saúde, bemestar e qualidade de vida dos idosos [6].

A literatura científica descreve evidências claras sobre o impacto negativo da IU sobre a QV, principalmente em mulheres e idosos jovens. Poucos são os estudos que evidenciam essa relação em longevos. Nesse contexto, o objetivo deste estudo foi observar a possível relação entre APS e IU, verificando o impacto da APS sobre a chance de desenvolver IU em longevos.

\section{MÉTODOS}

Trata-se de um estudo transversal e analítico. A técnica de amostragem foi de conglomerados representativos dos bairros de Porto Alegre, capital do Rio Grande do Sul, sendo selecionados idosos de 90 anos ou mais participantes do Projeto de Atenção Multiprofissional ao Longevo (AMPAL) da Pontifícia Universidade Católica do Rio Grande do Sul. Os participantes deveriam ter 90 anos ou mais, ser domiciliados em Porto Alegre e não residir em instituições de longa permanência, sendo incluídos idosos com qualquer nível funcional. O projeto é um estudo longitudinal aprovado pelo comitê de ética da PUCRS pelo número de parecer 1.639.961.

A escolha do corte etário de 90 anos deveu-se a experiências prévias dos pesquisadores, que encontraram em estudos anteriores e em publicações mais recentes, com dados da Pesquisa Nacional de Saúde de 2013, uma diferença significativa na funcionalidade e na relação funcionalidade e autopercepção de saúde entre nonagenários e as demais décadas etárias idosas a partir dos 60 anos [9]. Os nonagenários, também chamados centenariáveis, no Brasil, vêm ganhando destaque epidemiológico, pois menos de $10 \%$ conseguem se tornar centenários e é salientada a necessidade de uma atenção multiprofissional mais intensa [10].

A cidade de Porto Alegre está dividida em setores censitários através do sistema disponibilizado pelo ObservaPOA (2015). No site foi possível identificar os setores que tinham pelo menos seis longevos. Em seguida, grupos de alunos identificadores realizaram busca ativa dos possíveis longevos moradores desses locais. A partir dessa identificação criou-se no projeto uma lista de longevos distribuídos por setores, sendo a partir disso, sorteados para participar da pesquisa. A coleta dos dados iniciou em janeiro de 2016 e está em andamento.

A continência foi avaliada através do questionamento de perda urinária. A APS foi considerada a variável dependente do estudo e representada pelo autorrelato. A APS foi classificada em "boa ou ótima", "má ou péssima" e "regular".

As variáveis independentes consideradas de controle foram: sexo, idade (em anos), estado conjugal (casado, solteiro/divorciado e viúvo), presença de sintomas depressivos, e história de diagnóstico da doença, número de comorbidades, escore no Mini Exame do Estado Mental (MEEM) e percentual de facilidade no desempenho de atividades básicas e funcionais. A presença de sintomas depressivos foi avaliada pela Escala de Depressão Geriátrica 5 (GDS5) que consiste em cinco perguntas sobre a presença desses sintomas, sendo dois pontos a pontuação de corte [7]. Para as atividades básicas, os idosos foram 
questionados sobre a facilidade em banhar-se, vestirse, alimentar-se e ir ao banheiro, ao passo que, para as funcionais, foram investigadas a facilidade para caminhar quatro quadras, subir um lance de escada, carregar objetos de cinco quilos, levantar-se da cadeira sem usar as mãos, abaixar-se para pegar um objeto no chão, levantar os braços para cima da cabeça, agarrar objetos firmemente com as mãos e transferir-se de uma cama ou cadeira sozinhos. Cada atividade recebeu uma pontuação de acordo com o grau de facilidade, zero para quem não conseguia, um para quem relatava ser difícil, três mais ou menos fácil e, cinco, fácil. Do somatório dos pontos, 60 valor máximo e zero, mínimo, estabeleceu-se um cálculo simples, onde quem realizava com facilidade todas as atividades, alcançava, por exemplo, um percentual de $100 \%$.

O MEEM utilizado foi criado por Folstein, Folstein e Mchugh (1975) e encontra-se validado para idosos e traduzido para o idioma Português [8]. É composto de questões que avaliam as habilidades de linguagem, atenção e cálculo, memória imediata, orientação espacial e temporal, evocação, com pontuação variando de zero a 30 pontos e classificação cognitiva de acordo com a idade e a escolaridade. Para tanto, nesta análise utilizou-se apenas a pontuação total obtida no teste e a pontuação não foi utilizada como triagem cognitiva dos idosos com capacidade para responder aos questionários.

Foram realizados testes de Qui-quadrado para comparar as variáveis categóricas e realizados testes de Análise de Variância para verificar a presença de relação entre a variável dependente e as médias das variáveis numéricas independentes. Foram realizados testes de Regressão Logística Univariada para identificar as variáveis relacionadas com o risco de ter IU, de modo independente e testes de Regressão Logística Ajustada pela APS, para cada uma das demais variáveis, para investigar se a APS influenciava as demais variáveis investigadas na regressão. Foi utilizado o programa estatístico Epi Info ${ }^{\text {TM }} 7.2$ e considerados significativos valores de $\mathrm{p}<0,05$ e intervalos de confiança de $95 \%$ (IC95\%) maiores ou menores que 1 .

\section{RESULTADOS}

A Tabela 1 descreve as características sociodemográficas e de continência dos longevos entre os níveis de autopercepção de saúde. A frequência de APS teve relação significativa apenas com o diagnóstico prévio de depressão. Contudo é possível observar que, proporcionalmente, homens tinham maior frequência de relato de APS boa ou ótima, assim como os divorciados ou solteiros e os continentes (sem perda urinária).

A Tabela 2 apresenta as características sociodemográficas e clínicas numéricas dos longevos para os níveis de APS. Longevos com APS boa ou ótima apresentaram menor escore de sintomas depressivos e maiores percentuais de facilidade no desempenho de tarefas funcionais e básicas (respectivamente, $\mathrm{p}<0,001$, $\mathrm{p}=0,003$ e $\mathrm{p}=0,017$ ).

A Tabela 3 ilustra os resultados dos testes de regressão logística para a chance de apresentar IU relacionada às características sociodemográficas e clínicas de forma univariada e ajustada para APS. Observa-se que na regressão não ajustada, ser viúvo, ter maior frequência de diagnóstico de comorbidades e diagnóstico prévio de depressão aumentou a chance de ter IU, ao passo que, ser homem, apresentar maior percentual de facilidade em atividades básicas e

Tabela 1. Características sociodemográficas e de continência para as variáveis categóricas dos longevos entre os níveis de autopercepção de saúde.

\begin{tabular}{|c|c|c|c|c|}
\hline & APS boa ou ótima & APS regular & APS má ou péssima & p \\
\hline \multicolumn{5}{|l|}{ Sexo } \\
\hline Feminino & $65(50 \%)$ & $51(40 \%)$ & $13(10 \%)$ & 0,259 \\
\hline Masculino & $33(62 \%)$ & $16(30 \%)$ & $4(8 \%)$ & \\
\hline \multicolumn{5}{|l|}{ Estado conjugal } \\
\hline Casado & $24(60 \%)$ & $12(30 \%)$ & $4(10 \%)$ & 0,690 \\
\hline Solteiro/Divorciado & $13(62 \%)$ & $7(33 \%)$ & $1(5 \%)$ & \\
\hline Viúvo & $61(50 \%)$ & $48(40 \%)$ & $12(10 \%)$ & \\
\hline \multicolumn{5}{|l|}{ Perda de urina } \\
\hline Presença & $51(51 \%)$ & $41(41 \%)$ & $9(9 \%)$ & 0,484 \\
\hline Ausente & 47 (58\%) & $26(32 \%)$ & $8(10 \%)$ & \\
\hline Diagnóstico prévio de depressão & $18(31 \%)$ & $31(54 \%)$ & $9(16 \%)$ & $<0,001$ \\
\hline
\end{tabular}

APS, autopercepção de saúde. 
Tabela 2. Características sociodemográficas e clínicas numéricas dos longevos para os níveis de autopercepção de saúde.

\begin{tabular}{lcccc}
\hline & APS boa ou ótima & APS regular & APS má ou péssima & p \\
Idade & $92,8 \pm 3,79$ & $92,1 \pm 3,76$ & $91,4 \pm 3,03$ & 0,369 \\
GDS5 & $1,3 \pm 1,00$ & $1,8 \pm 1,21$ & $2,5 \pm 1,42$ & $<0,001$ \\
MEEM & $21,8 \pm 6,54$ & $20,3 \pm 7,19$ & $18,9 \pm 5,75$ & 0,151 \\
Comorbidades (número) & $4,4 \pm 2,03$ & $4,8 \pm 2,46$ & $5,7 \pm 2,80$ & 0,068 \\
Atividades funcionais & $62,6 \pm 24,93$ & $51,7 \pm 26,69$ & $44,8 \pm 16,89$ & 0,003 \\
Atividades básicas & $82,8 \pm 26,93$ & $71,4 \pm 31,31$ & $67,8 \pm 27,87$ & 0,017 \\
\hline
\end{tabular}

APS, autopercepção de saúde; GDS5, Escala de Depressão Geriátrica 5; MEEM, Mini Exame do Estado Mental.

Tabela 3. Regressão logística para a chance de apresentar incontinência urinária relacionada às características sociodemográficas e clínicas, univariada e ajustada para autopercepção de saúde.

\begin{tabular}{|c|c|c|c|c|c|c|}
\hline & \multicolumn{3}{|c|}{ Regressão não ajustada } & \multicolumn{3}{|c|}{ Regressão ajustada pela APS } \\
\hline & RC & IC $95 \%$ & $\mathbf{p}$ & RC & IC $95 \%$ & p \\
\hline \multicolumn{7}{|l|}{ Sexo (ref.: Feminino) } \\
\hline Masculino & 0,35 & $0,19-0,63$ & 0,001 & 0,37 & $0,19-0,71$ & 0,003 \\
\hline Idade & 1,04 & $0,96-1,12$ & 0,367 & 1,03 & $0,95-1,12$ & 0,461 \\
\hline \multicolumn{7}{|l|}{ Estado conjugal (ref.: Casado) } \\
\hline Solteiro/Divorciado & 1,81 & $0,71-4,66$ & 0,217 & 1,67 & $0,574,92$ & 0,349 \\
\hline Viúvo & 2,88 & $1,50-5,54$ & 0,002 & 3,18 & $1,506,73$ & 0,003 \\
\hline GDS5 & 1,15 & $0,92-1,44$ & 0,225 & 1,26 & $0,96-1,65$ & 0,095 \\
\hline MEEM & 0,94 & $0,90-0,98$ & 0,001 & 0,97 & $0,92-1,01$ & 0,122 \\
\hline Diagnóstico prévio de Depressão & 2,15 & $1,19-3,87$ & 0,011 & 1,77 & $0,90-3,50$ & 0,098 \\
\hline Comorbidades (número) & 1,14 & $1,01-1,29$ & 0,029 & 1,14 & $0,99-1,30$ & 0,062 \\
\hline Atividades funcionais & 0,98 & $0,97-0,99$ & $<0,001$ & 0,98 & $0,97-0,99$ & 0,004 \\
\hline Atividades básicas & 0,98 & $0,97-0,99$ & $<0,001$ & 0,98 & $0,97-1,00$ & 0,007 \\
\hline
\end{tabular}

APS, autopercepção de saúde; RC, razão de chances; GDS5, Escala de Depressão Geriátrica 5; MEEM, Mini Exame do Estado Mental.

funcionais e maior pontuação no MEEM diminuiu essa chance. Quando cada variável foi ajustada pela APS, o sexo masculino e a facilidade no desempenho de atividades básicas e funcionais continuaram sendo uma proteção e a viuvez um risco para desenvolver IU, enquanto a pontuação no MEEM, histórico de depressão e o número de comorbidades deixaram de ser significativos. Esses achados mostram que APS influenciou o efeito negativo do estado cognitivo avaliado pelo questionário e do número de comorbidades na chance de ter IU.

\section{DISCUSSÃO}

A APS é considerada um importante preditor de incapacidade funcional nos idosos. Em um estudo realizado com a base de dados da Pesquisa Nacional de Saúde de 2013, observou-se que a relação entre APS e atividades de vida diária foi mais diretamente proporcional entre os longevos, do que os idosos mais jovens, principalmente na relação entre APS e atividades instrumentais [9], o que vem ao encontro do estudo, no que se refere à significativa relação da APS com o percentual de facilidade em atividades básicas e funcionais.

Em relação a depressão e APS, um estudo envolvendo idosas evidenciou que, apesar de leve, a IU causou impacto negativo na vida das mesmas. Os autores sugerem que essa relação deve-se aos problemas de restrição social e alterações psicológicas decorrentes, visto que ocorre um desconforto com a perda de urina que pode levar ao isolamento social, diminuição da frequência de sair de casa e da autoestima [11], além de causar sentimentos de estresse, vergonha e sensação de incapacidade que age diretamente no dia a dia do idoso em sua qualidade de vida $[12,13]$.

O sexo feminino foi o mais acometido em nosso estudo pela IU. Isso vai ao encontro de um estudo que mostra que mulheres, pelo baixo nível de estrógeno 
após a menopausa, são mais acometidas por distúrbios urinários devido à diminuição da capacidade da bexiga, contribuindo para o aumento da frequência urinária e noctúria [14], além da anatomia da pelve feminina apresentar uma uretra mais curta, e ter os órgãos apoiados sobre a bexiga, causando uma maior compressão e levando ao aparecimento de IU [15].

O estado conjugal mais prevalente foi a viuvez. Uma pesquisa com moradores de uma instituição de longa permanência identificou características semelhantes, onde os incontinentes eram principalmente viúvos [16]. Outro estudo traz que, embora sem diferença significativa, os viúvos tinham mais frequência de fragilidade do que os demais estados conjugais [17].

Uma pesquisa mostrou que atividade física, principalmente de impacto, pode ser considerada fator de risco para o aparecimento de IU, devido ao impacto que a mesma exerce sobre o assolho pélvico, aumentando a pressão intra-abdominal [14]. Um estudo observou que a maioria dos idosos relatava uma boa APS, apesar do número de comorbidades. A hipótese dos autores era que a APS estaria mais relacionada com as incapacidades do que com a presença de doenças crônicas [18].

Foi encontrada uma menor APS em idosos com baixa escolaridade, e a justificativa seria que os idosos do estudo tinham dificuldade em acesso ao ambiente escolar no passado porque os atuais idosos viveram a infância numa época em que o ensino não era prioridade e atualmente esta realidade pode vir a afetar a realização das atividades e a APS [19]. A APS influenciou o efeito negativo do estado cognitivo avaliado pelo questionário. A literatura mostra que o resultado do MEEM em grupo de idosos incontinente apresentou pior déficit cognitivo. A IU influenciou a cognição, aumentando o percentual das associações com quadros demenciais, tornando-se uma das causas mais frequentes de asilamento e pior qualidade de vida [20].

No presente estudo, foram encontrados achados que mostram que a APS influenciou o efeito negativo de depressão e de ter IU, o que pode ser explicado por um estudo que sugere que a IU está relacionada com a autoestima diminuída, com a falta de controle sobre seu corpo, preocupação com a higiene, problemas psicológicos e afastamento da família e dos amigos. Podendo ocorrer isolamento social, depressão, tristeza, ansiedade e impacto na qualidade de vida [21].

Conclui-se que não houve relação estatisticamente significativa entre IU e APS, contudo, a APS influenciou no efeito das variáveis sociodemográficas e clínicas sobre a chance de ter IU. Uma hipótese possível é que a APS pode ser também um indicativo de tratamento. Assim, indivíduos que possuem comorbidades e têm a possibilidade de buscar tratamento, consequentemente poderão ter menos chances de apresentar IU. Com relação às atividades básicas e funcionais, as mesmas eram um fator protetor independente da APS, assim como o sexo masculino. A APS causou um impacto no histórico de depressão, no número de comorbidades e na pontuação no MEEM, resultando que deixassem de ter relação significativa com a IU.

\section{NOTAS}

\section{Apoio financeiro}

O presente trabalho foi realizado com apoio da Coordenação de Aperfeiçoamento de Pessoal de Nível Superior (CAPES) Código de Financiamento 001.

Declaração de conflito de interesses

Os autores declaram não haver conflitos de interesses relevantes ao conteúdo deste estudo.

Contribuições dos autores

Todos os autores fizeram contribuições substanciais para concepção, ou delineamento, ou aquisição, ou análise ou interpretação de dados; e redação do trabalho ou revisão crítica; e aprovação final da versão para publicação.

Disponibilidade dos dados e responsabilidade pelos resultados

Todos os autores declaram ter tido total acesso aos dados obtidos e assumem completa responsabilidade pela integridade destes resultados.

\section{REFERÊNCIAS}

1. Pereira LF, Lenardt MH, Michel T, Carneiro NHK, Bento, LF. Retrato do perfil de saúde-doença de idosos longevos usuários da atenção básica de saúde. Rev Enferm UERJ. 2015;23(5):649-55. https://doi.org/10.12957/reuerj.2015.5069

2. Kalache A. O mundo envelhece: é imperativo criar um pacto de solidariedade social. Cien Saude Colet. 2008;13(4): 1107-11. https://doi.org/10.1590/S1413-81232008000400002

3. Alvarenga-Martins N, Pinto PF, Arreguy-Sena C, Paschoalin HC, Moura DCA, Teixeira CV. Incontinência urinária: uma análise à luz das políticas de envelhecimento. Rev Enferm UFPE. 2017;11(3):1189-99.

4. Vecchia RD, Ruiz, T, Bocchi SCM, Corrente JE. Qualidade de vida na terceira idade: um conceito subjetivo. Rev Bras Epidemiol. 2005;8(3):246-52. https://doi.org/10.1590/S1415-790X2005000300006 
5. Pavão ALB, Werneck GL, Campos MR. Autoavaliação do estado de saúde e a associação com fatores sociodemográficos, hábitos de vida e morbidade na população: um inquérito nacional. Cad Saude Publica. 2013;29:723-34. https://doi. org/10.1590/S0102-311X2013000800010

6. Borges AM, Santos G, Kummer JÁ, Fior L, Dal Molin V, Wibelinger LM. Autopercepção de saúde em idosos residentes em um município do interior do Rio Grande do Sul. Rev Bras Geriatr Gerontol. 2014;17(1):79-86. https://doi.org/10.1590/ S1809-98232014000100009

7. Almeida MSC. Efetividade da escala de depressão geriátrica de cinco itens em população idosa da comunidade. 133f. 2010. Tese (Doutorado) - Pontifícia Universidade Católica do Rio Grande do Sul. Faculdade de Medicina. Programa de Pós-Graduação em Medicina e Ciências da Saúde. Porto Alegre: PUCRS, 2010.

8. Bertolucci PHF, Brucki SMD, Campacci SR, Juliano Y. O miniexame do estado mental em uma população geral: impacto da escolaridade. Arq Neuro-Psiquiatr. 1994; 52(1):1-7. https://doi.org/10.1590/S0004-282X1994000100001

9. Rocha JP, Oliveira GG, Jorge LB, Rodrigues FR, Morsch P, Bós AJG. Relação entre funcionalidade e autopercepção de saúde entre idosos jovens e longevos brasileiros. Rev Saúde Pesq. 2017;10(2):283-91. https://doi.org/10.17765/19831870.2017 v10n2p283-291

10. Camacho NCA, Morche KR, Muller ALW, Bós AJG. Por que nonagenários não se tornam centenários no Brasil? Rev AMRIGS. 2018;62(1):50-4.

11. Weschenfelder AJ, Strelow CS, Arruda GD, Froelich GTA, Pivetta HMF. Prevalência de incontinência urinária e seu impacto sobre a qualidade de vida de idosos: estudo comparativo entre meio urbano e meio rural. Rev Kairós. 2016;19(3): 67-77.

12. Bomfim IQM, Soutinho RSR, Araújo EM. Quality of life of women with urinary incontinence assisted in the public and private health system. UNOPAR Cient Ciênc Biol Saude. 2014;16(1):19-24.

13. Silva LWS, Lucas TQC, Santos SSOS, Novaes VS, Pires EPOR, Ludovici FMM. Physiotherapy urinary incontinence: views on the quality of life of elderly women. Rev Kairós. 2017;20(1):221-38.

14. Higa R, Lopes MHBM, Reis MJ. Fatores de risco para incontinência urinária na mulher. Rev Esc Enferm USP 2008; 42(1):187-92. https://doi.org/10.1590/S0080-62342008000100025

15. Neto ONV. Infecção do trato urinário. Rev Med Rib Preto. 2003;36(1):365-9.

16. Pitangui ACR, Silva AR, Araújo RC. Prevalência e impacto da incontinência urinária na qualidade de vida de idosas institucionalizadas. Rev Bras Geriatr Gerontol. 2012;15(4):619-26. https://doi.org/10.1590/S1809-98232012000400002

17. Paula AFM, Ribeiro LHMR, D’elboux MJ, Guariento ME. Avaliação da capacidade funcional, cognição e sintomatologia depressiva em idosos atendidos em ambulatório de Geriatria. Rev Bras Clin Med. 2013;11(3):212-8.

18. Poubel PB, Lemos ELC, Araujo FC, Leite GG, Freitas IS, Silva RMA, Borralho TG, Pires CAA. Self-perceived health and clinical-functional of elderly in a basic health unit in northern brazil. J. Health Biol Sci. 2017;5(1):71-78. https:// doi.org/10.12662/2317-3076jhbs.v5i1.1054.p71-78.2017

19. Güths FS, Jacob VM, Santos PV, Anziliero Arossi G, Umberto Béria J. Perfil sociodemográfico, aspectos familiares, percepção de saúde, capacidade funcional e depressão em idosos institucionalizados no Litoral Norte do Rio Grande do Sul, Brasil. Rev Bras Geriatr Gerontol. 2017;20(2):175-85. https://doi.org/10.1590/1981-22562017020.160058

20. Quadros LB, Aguiar A, Menezes AV, Alves EF, Nery T, Bezerra PP. Prevalência de incontinência urinária entre idosos institucionalizados e sua relação com o estado mental, independência funcional e comorbidades associadas. Acta Fisiatr. 2015;22(3):130-4.

21. Melo BES, Freitas BCR, Oliveira VRC, de Menezes RL. Correlação entre sinais e sintomas de incontinência urinária e autoestima em idosas. Rev Bras Geriatr Gerontol. 2012;15(1):41-50. https://doi.org/10.1590/S1809-982320120001 $00005 \epsilon$ 